\title{
Evidence to support natural hybridization between Anopheles sinensis and Anopheles kleini (Diptera: Culicidae): possibly a significant mechanism for gene introgression in sympatric populations
}

Wej Choochote ${ }^{1 * \dagger}$, Gi-Sik Min $^{2+}$, Pewpan M Intapan ${ }^{3,4}$, Chairat Tantrawatpan ${ }^{3,5}$, Atiporn Saeung ${ }^{1}$ and Viraphong Lulitanond ${ }^{3,6}$

\begin{abstract}
Background: Malaria caused by Plasmodium vivax is still a public health problem in the Republic of Korea (ROK), particularly regarding the recent re-emergence of this malarial species near the demilitarized zone in northwestern Paju City, Gyeonggi-do Province. Currently, at least 4 species (An. kleini, An. pullus, An. belenrae and An. lesteri) of the Hyrcanus Group are reported as possible natural vectors of vivax malaria in the ROK, and An. sinensis, which is the most dominant species, has long been incriminated as an important natural vector of this $P$. vivax. However, An. sinensis was ranked recently as a low potential vector. According to the discovery of natural hybrids between $A n$. sinensis (a low potential vector for P. vivax) and An. kleini (a high potential vector for P. vivax) in Paju City, intensive investigation of this phenomenon is warranted under laboratory conditions.
\end{abstract}

Methods: Mosquitoes were collected during 2010-2012 from Paju City, ROK. Hybridization experiments used iso-female line colonies of these anophelines together with DNA analysis of ribosomal DNA [second internal transcribed spacer (ITS2)] and mitochondrial DNA [cytochrome c oxidase subunit I (COI)] of the parental colonies, $\mathrm{F}_{1}$-hybrids and repeated backcross progenies were performed intensively by using a PCR-based assay and pyrosequencing technology.

Results: The results from hybridization experiments and molecular investigations revealed that the mitochondrial $\mathrm{COl}$ gene was introgressed from An. sinensis into An. kleini. The An. sinensis progenies obtained from consecutive repeated backcrosses in both directions, i.e., $F_{2^{-} 11}$ progeny [(An. sinensis $\times$ An. kleini) $\times$ An. sinensis $]$ and $\mathrm{F}_{3^{-} 5}$ progeny [(An. Kleini $\times$ An. sinensis) $\times$ An. kleini] provided good supportive evidence.

Conclusions: This study revealed introgression of the mitochondrial COI gene between An. sinensis and An. kleini through consecutive repeated backcrosses under laboratory conditions. This new body of knowledge will be emphasized in reliable promising strategies in order to replace the population of An. Kleini as a high potential vector for $P$. vivax, with that of a low potential vector, An. sinensis, through the mechanism of gene introgression in nature.

Keywords: Anopheles sinensis, An. kleini, Hybridization experiment, Second internal transcribed spacer, Cytochrome c oxidase subunit I, Introgression

\footnotetext{
* Correspondence: wchoocho@mail.med.cmu.ac.th

${ }^{\dagger}$ Equal contributors

'Department of Parasitology, Faculty of Medicine, Chiang Mai University,

Chiang Mai 50200, Thailand

Full list of author information is available at the end of the article
}

C Biomed Central

(c) 2014 Choochote et al.; licensee BioMed Central Ltd. This is an Open Access article distributed under the terms of the Creative Commons Attribution License (http://creativecommons.org/licenses/by/2.0), which permits unrestricted use, distribution, and reproduction in any medium, provided the original work is properly cited. The Creative Commons Public Domain Dedication waiver (http://creativecommons.org/publicdomain/zero/1.0/) applies to the data made available in this article, unless otherwise stated. 


\section{Background}

Up until now, at least 26 species members of the Anopheles hyrcanus group have been reported, and their distribution has extended widely from Europe to East and Southeast Asia, including some of the off-lying islands of the Indian and Pacific Oceans [1]. Some species of the Hyrcanus Group are accepted as important vectors in transmitting human diseases, e.g., malaria (Plasmodium vivax) [2-14], filariae (Wuchereria bancrofti and Brugia malayi) [15,16], and Japanese encephalitis virus [17,18], particularly in the Oriental and contiguous parts of eastern Palaearctic regions.

At least 6 species (Anopheles sinensis, An. lesteri, An. pullus, An. sineroides, An. belenrae and An. kleini) of the Hyrcanus Group are found indigenously in the Republic of Korea (ROK). Among these, An. sinensis has long been incriminated as the most dominant and important natural vector of $P$. vivax, especially due to the recent reemergence of vivax malaria near the demilitarized zone in northwestern Paju City, Gyeonggi-do Province [3,19-22]. However, the low concentration of circumsporozoite (CS) antigen obtained from wild-caught females [6], and very low sporozoite rates recovered from laboratory susceptibility tests [7,9], have brought about the cryptic status of An. sinensis as a natural vector of vivax malaria transmission in the ROK. Consequently, the implication of other An. hyrcanus species, i.e., An. kleini, An. pullus, An. belenrae and $A n$. lesteri as possible natural vectors of vivax malaria in the ROK has been proposed extensively [8,9], even though the latter species is thought to have a small population [7]. Remarkably, An. sinensis strain from China has been incriminated recently as an efficient vector of P. vivax [11].

The discovery of natural hybrids has been reported from some important malaria vectors, for instance, between An. gambiae and An. arabiensis [23], An. scanloni (= dirus C) and An. baimaii (= dirus D) [24], and An. minimus and An. harrisoni [25,26]. Regarding An. kleini (a high potential vector for $P$. vivax) and An. sinensis (a low potential vector for $P$. vivax) [7,9], a single gravid, natural hybrid female was discovered between these 2 anopheline species from a total of 658 wild-caught females in Paju City, ROK; an endemic zone of vivax malaria from 2004 to 2008. These wild-caught females comprised 360 An. sinensis, 258 An. pullus, 20 An. belenrae, 15 An. kleini, 3 An. sineroides, 1 An. lesteri and 1 hybrid female between An. kleini and An. sinensis [27]. The results of self-crossing between $\mathrm{F}_{1}$-progenies derived from one egg-batch of an egg-laid gravid female, and molecular investigations, revealed possible natural backcrossing (introgression) between a hybrid female and male $A n$. sinensis. There is no clear basic information on the introgression between $A n$. sinensis and An. kleini, as proposed by [27]. Thus, systematic investigations into the role of introgressive hybridization between these two anopheline species were performed in this study. Accordingly, attempts were made to establish iso-female line colonies of $A n$. sinensis and An. kleini, perform crossing experiments (reciprocal and back crosses), investigations of reproductive systems of hybrid and backcross progenies, and compare DNA analysis of ribosomal DNA (ITS2) and mitochondrial DNA (COI) of the parental, $\mathrm{F}_{1}$-hybrids with that of repeated backcross progenies by using a PCR-based assay and pyrosequencing technology.

\section{Methods}

\section{Establishment of iso-female lines}

Mosquitoes were collected during 2010-2012 from Paju City, Republic of Korea (ROK), by placing a light trap in cowsheds. Then, wild-caught females were transported for colonization to the insectary of the Department of Parasitology, Faculty of Medicine, Chiang Mai University, Chiang Mai, Thailand. Four iso-female lines of both $A n$. sinensis and $A n$. kleini were established successfully using the methods of [28]. An $F_{1}$-progeny of each iso-female line was used for species identification following the keys of [29] as well as a molecular assay [30]. Then, one isofemale line of each species, with molecular identification of both nuclear (ITS2) and mitochondrial (COI) genes, were well matched with those in the GenBank nucleotide sequence database, and selected, i.e., An. sinensis $\mathrm{F}_{0}-1$ (SF0-1) and An. kleini $\mathrm{F}_{0}-1$ (KF0-1). These iso-female lines have been maintained in colonies in the laboratory at Chiang Mai University for more than 10 consecutive generations, and used for hybridization experiments and comparative DNA sequence analyses.

\section{Hybridization experiments}

Hybridization experiments (reciprocal and back crosses, and repeated backcross progenies) between An. sinensis and $A n$. kleini were performed by using virgin females and males and following the techniques previously reported by [31]. Post-mating reproductive isolation was recorded using the criteria of low viability (hatchability, survival, pupation, and emergence), adult sex distortion and abnormal morphology of the reproductive system.

\section{PCR identification, dideoxy sequencing and phylogenetic analysis}

DNA was extracted individually from 60 mosquitoes using the RED Extract-N-Amp ${ }^{\text {Tm }}$ Tissue PCR kit (Sigma-Aldrich, Spruce Street, SL) as shown in Table 1. Primers for the amplification of ITS2 and COI regions followed a previous report by [30]. The ITS2 region of the rDNA was amplified using the ITS2 Forward and ANO 28S-20 primers $[30,32]$. The mitochondrial COI gene was amplified using the LCO1490 (5'-GGT CAA CAA ATC ATA AAG ATA TTG G-3') and HCO2198 (5'-TAA ACT TCA GGG 
Table 1 Species identification of An. sinensis and An. kleini samples based on ITS2 and COI sequences, and their GenBank accession numbers

\begin{tabular}{|c|c|c|c|c|}
\hline \multirow[t]{2}{*}{ Samples (Female x Male) } & \multirow[t]{2}{*}{ Code of samples } & \multicolumn{2}{|c|}{ Identified species name (GenBank accession number) } & \multirow[t]{2}{*}{ Reference } \\
\hline & & ITS2 & $\mathrm{COI}$ & \\
\hline \multicolumn{5}{|l|}{ Parental } \\
\hline An. sinensis $F_{0}-1$ & SF0-1 & An. sinensis (KC797396) & An. sinensis (KC797435) & This study \\
\hline An. sinensis $F_{0}-2$ & SF0-2 & An. sinensis (KC797397) & An. sinensis (KC797436) & This study \\
\hline An. sinensis $\mathrm{F}_{0}-3$ & SFO-3 & An. sinensis (KC797398) & An. sinensis (KC797437) & This study \\
\hline An. sinensis $F_{0}-4$ & SF0-4 & An. sinensis (KC797399) & An. sinensis (KC797438) & This study \\
\hline An. kleini $\mathrm{F}_{0}-1$ & KF0-1 & An. kleini (KC797431) & An. kleini (KC797439) & This study \\
\hline An. kleini $\mathrm{F}_{0}-2$ & KFO-2 & An. kleini (KC797432) & An. sinensis (KC797440) & This study \\
\hline An. kleini $\mathrm{F}_{0}-3$ & KFO-3 & An. kleini (KC797433) & An. sinensis (KC797441) & This study \\
\hline An. Kleini $\mathrm{F}_{0}-4$ & KFO-4 & An. kleini (KC797434) & An. sinensis (KC797442) & This study \\
\hline \multicolumn{5}{|l|}{ Reciprocal crosses } \\
\hline An. sinensis $\times$ An.kleini $\mathrm{F}_{1-1}$ & SKF1-1 & Mixed & An. sinensis (KC797446) & This study \\
\hline An. sinensis $\times$ An.kleini $\mathrm{F}_{1}-2$ & SKF1-2 & Mixed & An. sinensis (KC797447) & This study \\
\hline An. sinensis $\times$ An.kleini $F_{1}-3$ & SKF1-3 & Mixed & An. sinensis (KC797448) & This study \\
\hline An. kleini $\times$ An. sinensis $\mathrm{F}_{1-1}$ & KSF1-1 & Mixed & An. sinensis (KC797443) & This study \\
\hline An. kleini $\times$ An. sinensis $F_{1}-2$ & KSF1-2 & Mixed & An. kleini (KC797444) & This study \\
\hline An. kleini $\times$ An. sinensis $F_{1}-3$ & KSF1-3 & Mixed & An. sinensis (KC797445) & This study \\
\hline
\end{tabular}

Back crosses

(An. sinensis $\times$ An. kleini) $\times$ An. sinensis hybridF ${ }_{1} \times$ sinensis- $1=>\left(\right.$ hybridF $\left._{2}-1\right)$ hybridF $F_{1} \times$ sinensis- $2=>\left(\right.$ hybridF $\left._{2}-2\right)$

SKSF2-1

SKSF2-2

hybridF ${ }_{1} \times$ sinensis- $=>\left(\right.$ hybridF $\left._{2}-3\right)$

hybridF $2 \times$ sinensis- $1=>\left(\right.$ hybridF $\left._{3}-1\right)$

hybridF $F_{2} \times$ sinensis-2 $=>\left(\right.$ hybridF $\left._{3}-2\right)$

hybridF $2 \times$ sinensis-3 $=>\left(\right.$ hybridF $\left._{3}-3\right)$

hybridF ${ }_{3} \times$ sinensis- $1=>\left(\right.$ hybridF $\left._{4}-1\right)$

hybridF $F_{3} \times$ sinensis- $2=>\left(\right.$ hybridF $\left._{4}-2\right)$

hybridF $F_{3} \times$ sinensis-3 $=>\left(\right.$ hybridF $\left._{4}-3\right)$

hybridF $_{4} \times$ sinensis- $1=>\left(\right.$ hybridF $\left._{5}-1\right)$

hybridF 4 × sinensis- $2=>\left(\right.$ hybridF $\left._{5}-2\right)$

hybridF $_{4} \times$ sinensis-3 $=>$ (hybridF $\left._{5}-3\right)$

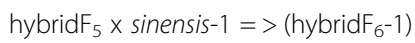

hybridF $F_{5} \times$ sinensis- $2=>\left(\right.$ hybridF $\left._{6}-2\right)$

hybridF $_{5} \times$ sinensis-3 $=>$ (hybridF $_{6}-3$ )

hybridF $_{6} \times$ sinensis $-1^{-1}=$ (hybridF $\left._{7}-1\right)$

hybridF $_{6} \times$ sinensis- $2=>\left(\right.$ hybridF $\left._{7}-2\right)$

hybridF $_{6} \times$ sinensis $-3=>\left(\right.$ hybridF $\left._{7}-3\right)$

hybridF $_{7} \times$ sinensis-1 $=>\left(\right.$ hybridF $\left._{8}-1\right)$

hybridF $_{7} \times$ sinensis- $2=>\left(\right.$ hybridF $\left._{8}-2\right)$

hybridF $_{7} \times$ sinensis $-3=>$ (hybridF $_{8}-3$ )

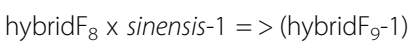

hybridF ${ }_{8} \times$ sinensis- $2=>\left(\right.$ hybridF $\left._{9}-2\right)$

hybridF ${ }_{8} \times$ sinensis-3 $=>$ (hybridF $_{9}-3$ )

\section{SKSF2-3}

SKSF3-1

SKSF3-2

SKSF3-3

SKSF4-1

SKSF4-2

SKSF4-3

SKSF5-1

SKSF5-2

SKSF5-3

SKSF6-1

SKSF6-2

SKSF6-3

SKSF7-1

SKSF7-2

SKSF7-3

SKSF8-1

SKSF8-2

SKSF8-3

SKSF9-1

SKSF9-2

SKSF9-3
Mixed

Mixed

Mixed

An. sinensis (KC797400)

An. sinensis (KC797401)

An. sinensis (KC797402)

An. sinensis (KC797403)

An. sinensis (KC797404)

An. sinensis (KC797405)

An. sinensis (KC7974006)

An. sinensis (KC797407)

An. sinensis (KC797408)

An. sinensis (KC797409)

An. sinensis (KC797410)

An. sinensis (KC797411)

An. sinensis (KC797412)

An. sinensis (KC797413)

An. sinensis (KC797414)

An. sinensis (KC797415)

An. sinensis (KC797416)

An. sinensis (KC797417)

An. sinensis (KC797418)

An. sinensis (KC797419)

An. sinensis (KC797420)
An. sinensis (KC797449)

An. sinensis (KC797450)

An. sinensis (KC797451)

An. sinensis (KC797452)

An. sinensis (KC797453)

An. sinensis (KC797454)

An. sinensis (KC797455)

An. sinensis (KC797456)

An. sinensis (KC797457)

An. sinensis (KC797458)

An. sinensis (KC797459)

An. sinensis (KC797460)

An. sinensis (KC797461)

An. sinensis (KC797462)

An. sinensis (KC797463)

An. sinensis (KC797464)

An. sinensis (KC797465)

An. sinensis (KC797466)

An. sinensis (KC797467)

An. sinensis (KC797468)

An. sinensis (KC797469)

An. sinensis (KC797470)

An. sinensis (KC797471)

An. sinensis (KC797472)
This study

This study

This study

This study

This study

This study

This study

This study

This study

This study

This study

This study

This study

This study

This study

This study

This study

This study

This study

This study

This study

This study

This study

This study 
Table 1 Species identification of An. sinensis and An. kleini samples based on ITS2 and COI sequences, and their GenBank accession numbers (Continued)

\begin{tabular}{|c|c|c|c|c|}
\hline hybridF $_{9} \times$ sinensis- $1=>\left(\right.$ hybridF $\left._{10^{-1}}\right)$ & SKSF10-1 & An. sinensis (KC797421) & An. sinensis (KC797473) & This study \\
\hline hybridF $_{9} \times$ sinensis $-2=>\left(\right.$ hybridF $\left._{10}-2\right)$ & SKSF10-2 & An. sinensis (KC797422) & An. sinensis (KC797474) & This study \\
\hline hybridF $_{9} \times$ sinensis $-3=>\left(\right.$ hybridF $\left._{10}-3\right)$ & SKSF10-3 & An. sinensis (KC797423) & An. sinensis (KC797475) & This study \\
\hline hybridF $_{10} \times$ sinensis- $1=>\left(\right.$ hybridF $\left.F_{11}-1\right)$ & SKSF11-1 & An. sinensis (KC797424) & An. sinensis (KC797476) & This study \\
\hline hybridF $_{10} \times$ sinensis- $2=>\left(\right.$ hybridF $\left.{ }_{11}-2\right)$ & SKSF11-2 & An. sinensis (KC797425) & An. sinensis (KC797477) & This study \\
\hline hybridF $_{10} \times$ sinensis-3 $=>\left(\right.$ hybridF $\left._{11}-3\right)$ & SKSF11-3 & An. sinensis (KC797426) & An. sinensis (KC797478) & This study \\
\hline hybridF $_{11} \times$ hybridF $_{11^{-1}}$ & HF11-1 & An. sinensis (KC797427) & An. sinensis (KC797479) & This study \\
\hline hybridF $_{11} \times$ hybridF $_{11}-2$ & HF11-2 & An. sinensis (KC797428) & An. sinensis (KC797480) & This study \\
\hline hybridF $_{11} \times$ hybridF $_{11}-3$ & HF11-3 & An. sinensis (KC797429) & An. sinensis (KC797481) & This study \\
\hline hybridF $_{11} \times$ hybridF $_{11}-4$ & HF11-4 & An. sinensis (KC797430) & An. sinensis (KC797482) & This study \\
\hline \multicolumn{5}{|l|}{ Back crosses } \\
\hline \multicolumn{5}{|l|}{ (An. kleini $\times$ An. sinensis) $\times$ An. kleini } \\
\hline hybridF $_{1} \times$ kleini-1 $=>$ (hybridF $\left._{2}-1\right)$ & KSKF2-1 & An. kleini (KC890843) & An. kleini (KC797483) & This study \\
\hline hybridF $_{1} \times$ kleini- $2=>\left(\right.$ hybridF $\left._{2}-2\right)$ & KSKF2-2 & An. kleini (KC890844) & An. kleini (KC797484) & This study \\
\hline hybridF $_{1} \times$ kleini-3 $=>$ (hybridF $_{2}-3$ ) & KSKF2-3 & An. kleini (KC890845) & An. kleini (KC797485) & This study \\
\hline hybridF $_{2} \times$ kleini- $1=>\left(\right.$ hybridF $\left._{3}-1\right)$ & KSKF3-1 & An. kleini (KC890846) & An. sinensis (KC797486) & This study \\
\hline hybridF $_{2} \times$ kleini-2 $=>\left(\right.$ hybridF $\left._{3}-2\right)$ & KSKF3-2 & An. kleini (KC890847) & An. sinensis (KC797487) & This study \\
\hline hybridF $_{2} \times$ kleini-3 $=>$ (hybridF ${ }_{3}-3$ ) & KSKF3-3 & An. Kleini (KC890848) & An. sinensis (KC797488) & This study \\
\hline hybridF $_{3} \times$ kleini- $1=>\left(\right.$ hybridF $\left._{4^{-1}}\right)$ & KSKF4-1 & An. kleini (KC890849) & An. sinensis (KC797489) & This study \\
\hline hybridF $_{3} \times$ kleini-2 $=>\left(\right.$ hybridF $\left._{4}-2\right)$ & KSKF4-2 & An. Kleini (KC890850) & An. sinensis (KC797490) & This study \\
\hline hybridF $_{3} \times$ kleini-3 $=>\left(\right.$ hybridF $\left._{4}-3\right)$ & KSKF4-3 & An. kleini (KC890851) & An. sinensis (KC797491) & This study \\
\hline hybridF $_{4} \times$ kleini-1 $=>\left(\right.$ hybridF $\left._{5}-1\right)$ & KSKF5-1 & An. kleini (KC890852) & An. sinensis (KC797492) & This study \\
\hline hybridF $_{4} \times$ kleini-2 $=>\left(\right.$ hybridF $\left._{5}-2\right)$ & KSKF5-2 & An. kleini (KC890853) & An. sinensis (KC797493) & This study \\
\hline hybridF $_{4} \times$ kleini-3 $=>\left(\right.$ hybridF $\left._{5}-3\right)$ & KSKF5-3 & An. kleini (KC890854) & An. sinensis (KC797494) & This study \\
\hline \multirow[t]{2}{*}{ An. sinensis } & - & An. sinensis (GU384700) & - & {$[30]$} \\
\hline & & - & An. sinensis (AY444351) & [39] \\
\hline \multirow[t]{2}{*}{ An. kleini } & - & An. kleini (GU384713) & - & {$[30]$} \\
\hline & & - & An. kleini (GQ265917) & {$[27]$} \\
\hline
\end{tabular}

TGA CCA AAA AAT CA-3') primers of [33]. PCR reaction was carried out in a total volume of $25 \mu \mathrm{l}$ containing $10 \mathrm{pM}$ of each primer; and $2.5 \mu \mathrm{l}$ of $10 \mathrm{X}$ buffer containing $50 \mathrm{mM} \mathrm{KCl,} 10 \mathrm{mM}$ Tris-HCI, 0.1\% Triton X 100 supplemented with $1.5 \mathrm{mM} \mathrm{MgCl}_{2}$ (Promega, USA), $200 \mu \mathrm{M}$ of each dNTP (GeneCraft, Germany), $0.5 \mu \mathrm{l}$ of Taq DNA polymerase (Promega, USA) and 10-100 pg of genomic DNA. The amplification profile comprised initial denaturation at $94^{\circ} \mathrm{C}$ for $3 \mathrm{~min}$, with 30 cycles at $94^{\circ} \mathrm{C}$ for $30 \mathrm{sec}$, $55^{\circ} \mathrm{C}$ for $30 \mathrm{sec}$, and $72^{\circ} \mathrm{C}$ for $2 \mathrm{~min}$, and a final extension at $72^{\circ} \mathrm{C}$ for $7 \mathrm{~min}$. The PCR products were separated by electrophoresis on a $1.5 \%$ agarose gel stained with ethidium bromide. Finally, the purified PCR products were subjected to sequencing in an ABI PRISM 3700 DNA Analyzer (Applied Biosystems, Foster City, CA) using a Dye Terminator Cycle Sequencing Ready Reaction Kit (Applied Biosystems). The sequence data obtained were deposited in the GenBank nucleotide sequence database
(Table 1). The newly determined ITS2 and COI sequences were also compared with those available in GenBank, using the Basic Local Alignment Search Tool (BLAST) available at http://blast.ncbi.nlm.nih.gov/Blast.cgi. The DNA sequence data were edited manually in BioEdit version 7.0.5.3 [34] and aligned using CLUSTAL W [35]. Constructions of neighbor-joining trees using distance [36], and the bootstrap test with 1,000 replications, were performed with the MEGA version 4.0 program based on COI sequences [37]. The COI sequences of An. peditaeniatus (GenBank accession number AB539069) [38] and An. pullus (GenBank accession number AB444348) [39] were included in phylogenetic analysis.

\section{PCR and pyrosequencing for the detection of An. sinensis} and An. kleini sequences

DNA was extracted from each adult female mosquito of $A n$. sinensis and An. kleini using a NucleoSpin tissue kit 
(Macherey-Nagel GmbH and Co., Duren, Germany). The procedure for the pyrosequencing assay followed that previously described by [40]. The forward primer (Anop_COI_F: 5'-GAG CCC CTG ATA TAG CTT TTC CT-3'), and biotinylated reverse primers (Anop_COI_Rb: 5' - Biotin-CCA GAT GAA AGT GGG GGA TAA -3'), were designed to amplify a 142-bp fragment of COI, and a primer Anop_COI_S (5'-ATA AGT TTT TGA ATA TTA CC -3') for pyrosequencing. Positivecontrol plasmids of each species were constructed by amplification of the 142-bp PCR products using Anop_COI_F and Anop_COI_R primers. They were ligated and transformed into a pGEM -T Easy vector (Promega, WI) and an Escherichia coli JM109, respectively. The recombinant plasmids were sequenced bidirectionally in order to confirm the correction of data. The $142 \mathrm{bp}$ was amplified from genomic DNA using the Anop_COI_F and Anop_COI_Rb primers. The reactions of PCR amplification were performed in a total volume of $25 \mu \mathrm{l}$ containing 1X PCR buffer (Invitrogen, Carlsbad, CA) with $0.2 \mathrm{mM}$ of each dNTP, $2 \mathrm{mM} \mathrm{MgSO} 4,0.4 \mu \mathrm{M}$ of each primer, $0.625 \mathrm{U}$ of Platinum Taq DNA polymerase high fidelity (Invitrogen, Carlsbad, CA) and $2 \mu \mathrm{l}$ of the DNA sample. The PCR assay was conducted using a GeneAmp PCR system 9700 thermal cycler (Applied Biosystems, Singapore). The thermocycling program included: initial denaturation at $94^{\circ} \mathrm{C}$ for $5 \mathrm{~min}$, with 40 cycles at $94^{\circ} \mathrm{C}$ for $30 \mathrm{sec}, 57^{\circ} \mathrm{C}$ for $30 \mathrm{sec}$, and $72^{\circ} \mathrm{C}$ for $30 \mathrm{sec}$, and a final extension at $72^{\circ} \mathrm{C}$ for $7 \mathrm{~min}$. PCR amplicons were detected by electrophoresis on $1.5 \%$ agarose gels. For pyrosequencing assays, $20 \mu \mathrm{l}$ of the biotinylated PCR product of each sample was immobilized in the binding buffer with Streptavidin Sepharose ${ }^{\mathrm{TM}}$ beads (GE Healthcare BioSciences AB, Uppsala, Sweden). The beads together with the DNA were aspirated to a 96 format filter tool and passed through $70 \%$ ethanol and $0.2 \mathrm{M} \mathrm{NaOH}$, and then washed with $10 \mathrm{mM}$ Tris-acetate $(\mathrm{pH} 7.6)$ using a PyroMark ${ }^{\mathrm{TM}}$ Vacuum Prep Workstation (Biotage, Uppsala, Sweden). The beads were released subsequently into a $\mathrm{PSQ}^{\mathrm{TM}} 96$ plate low (Biotage) containing $0.4 \mu \mathrm{M}$ Anop_COI_S sequencing primer in the annealing buffer. The samples were heated to $80^{\circ} \mathrm{C}$ for $2 \mathrm{~min}$ before performing pyrosequencing reactions using PyroMark ${ }^{\mathrm{TM}}$ Gold Q96 SQA reagents and subjecting them to the PyroMark $^{\text {тм }}$ Q96 ID instrument (Biotage). Positive and negative controls were included in each pyrosequencing assay. Finally, the PyroMark ${ }^{\mathrm{Tm}}$ Q96 ID software version 1.0 was used to analyze the results and generate a pyrogram.

\section{Results}

\section{Hybridization experiments}

The hatchability, pupation, emergence and adult sexratio of parental, reciprocal and back crosses, repeated backcrosses and hybrid crosses between An. sinensis and An. kleini were 88.06-92.57\%, 77.96-92.97\%, 94.10-96.98\% and $0.81-0.89 ; 82.94-85.07 \%, 98.00-100 \%, 98.87-100 \%$ and 1.04-1.38; 61.10-69.01\%, 100\%, 97.10-100\% and 1.13-1.43; $66.00-86.61 \%, 87.87-100 \%, 90.07-100 \%$ and $0.96-1.38$, and 95.14\%, 91.05\%, 84.91\% and 0.94; respectively (Table 2). All crosses yielded viable progenies, with no evidence of genetic incompatibility being observed among them, except for only sterile $\mathrm{F}_{1}$-progeny males of which the atrophy of testes and accessory glands were recovered from the reciprocal crosses in both directions (An. sinensis $\mathrm{x}$ An. kleini and An. kleini x An. sinensis) (Figure 1A), while normal development occurred in all males from repeated backcrosses (Figure 1B). On the other hand, all females from $\mathrm{F}_{1}$-hybrids and repeated backcrosses yielded normal development of ovarian follicles (Figures $1 \mathrm{C}$ and D). Regarding repeated backcross groups, the experiments of $($ An. sinensis $\mathrm{x} A n$. kleini $) \times A n$. sinensis were carried out from $\mathrm{F}_{1-10}$, whereas those of $(A n$. kleini $\times A n$. sinensis $) \times$ $A n$. kleini were investigated from $\mathrm{F}_{1-5}$. The reason for this was that the repeated backcross of $(A n$. kleini $\times A n$. sinensis $) \times A n$. kleini could be carried out in only the fifth generation, which led to a lack of hybrids for further experimentation (repeated twice). Regarding hybrid crosses, the experiment was performed only on the $\mathrm{F}_{11}$ of $[(A n$. sinensis $\times A n$. kleini $) \times A n$. sinensis $] \times[(A n$. sinensis $\times$ An. kleini) $\times$ An. sinensis $]$.

\section{PCR species identification}

For molecular identification, sequences of nuclear ITS2 and mitochondrial COI genes were determined for both the parent mosquitoes and their hybrid progenies. Sequences of $A n$. sinensis parents $\left(\mathrm{F}_{0}\right)$ were well matched in both genes with those in the GenBank nucleotide sequence database. The four specimens of an An. kleini parent $\left(\mathrm{F}_{0}\right)$ were also checked, and they all matched $A n$. kleini based on ITS2 sequences. However, their COI gene, $A n$. kleini matched in both species, with 1 being An. kleini and 3 An. sinensis (Table 1).

The $\mathrm{F}_{1}$-hybrid progenies have mixed sequences in their nuclear ITS2 gene. They have heterogeneous ITS2 sequences because they receive ribosomal RNA genes, including ITS2 from both parents. As for a maternal, mitochondrial COI gene, the hybrids followed the trait of the mother. All the progenies of An. sinensis $\times$ An. kleini matched $A n$. sinensis based on COI sequences. However, progenies of $A n$. kleini $\times A n$. sinensis matched both $A n$. kleini and $A n$. sinensis based on COI sequences, in which two progenies matched An. sinensis and one An. kleini (Table 1).

Two backcrossing groups were checked, with one being a progeny of $(A n$. sinensis $\times A n$. kleini $) \times A n$. sinensis and the other a progeny of $(A n$. kleini $\times A n$. sinensis $) \times$ An. kleini. In the $(A n$. sinensis $\times A n$. kleini $) \times A n$. 
Table 2 Hybridization experiments between isolines of An. sinensis and An. kleini

\begin{tabular}{|c|c|c|c|c|c|c|c|}
\hline \multirow[t]{2}{*}{ Crosses (Female $x$ Male) } & \multirow{2}{*}{$\begin{array}{l}\text { Total eggs } \\
\text { (number) }\end{array}$} & \multirow{2}{*}{$\begin{array}{c}\text { Embryonation } \\
\text { rate }^{\dagger}\end{array}$} & \multirow{2}{*}{$\begin{array}{l}\text { Hatched } \\
\text { n (\%) }\end{array}$} & \multirow{2}{*}{$\begin{array}{l}\text { Pupation } \\
\text { n (\%) }\end{array}$} & \multirow{2}{*}{$\begin{array}{c}\text { Emergence } \\
\mathrm{n}(\%)\end{array}$} & \multicolumn{2}{|c|}{ Total emergence $\mathrm{n}(\%)$} \\
\hline & & & & & & Female & Male \\
\hline \multicolumn{8}{|l|}{ Parental crosses } \\
\hline An. sinensis $\times$ An. sinensis & $538(258,280)$ & 96 & $498(92.57)$ & $463(92.97)$ & 449 (96.98) & $211(46.99)$ & $238(53.01)$ \\
\hline An. kleini x An. kleini & $469(263,206)$ & 89 & $413(88.06)$ & $322(77.96)$ & $303(94.10)$ & $136(44.88)$ & $167(55.12)$ \\
\hline \multicolumn{8}{|l|}{ Reciprocal crosses } \\
\hline An. sinensis $\times$ An. kleini & $529(288,241)$ & 86 & $450(85.07)$ & $441(98.00)$ & $436(98.87)$ & $253(58.03)$ & $183(41.97)^{\dagger+}$ \\
\hline An. kleini $\times$ An. sinensis & $422(239,183)$ & 83 & $350(82.94)$ & $350(100.00)$ & $350(100.00)$ & $178(50.86)$ & $172(49.14)^{\dagger \dagger}$ \\
\hline \multicolumn{8}{|l|}{ Back crosses } \\
\hline$\left(\right.$ An. sinensis $\times$ An. kleini) $\mathrm{F}_{1} \times$ An. sinensis & $401(218,183)$ & 63 & $245(61.10)$ & $245(100.00)$ & $238(97.10)$ & $140(58.82)$ & $98(41.18)$ \\
\hline$\left(\right.$ An. sinensis $\times$ An. kleini) $\mathrm{F}_{2} \times$ An. sinensis & $397(207,190)$ & 88 & $332(83.67)$ & 309 (93.07) & $309(100.00)$ & $173(55.96)$ & $136(44.04)$ \\
\hline$\left(\right.$ An. sinensis $\times$ An. kleini) $F_{3} \times$ An. sinensis & $386(211,175)$ & 74 & $282(73.05)$ & $265(93.97)$ & $265(100.00)$ & $143(53.96)$ & $122(46.04)$ \\
\hline$\left(\right.$ An. sinensis $\times$ An. kleini) $\mathrm{F}_{4} \times$ An. sinensis & $413(240,173)$ & 79 & $319(77.24)$ & $284(89.02)$ & $259(91.20)$ & $137(52.90)$ & $122(47.10)$ \\
\hline (An. sinensis $\times$ An. kleini) $F_{5} \times$ An. sinensis & $412(232,180)$ & 69 & $282(68.45)$ & $282(100.00)$ & $254(90.07)$ & $139(54.72)$ & $115(45.28)$ \\
\hline (An. sinensis $\times$ An. kleini) $\mathrm{F}_{6} \times$ An. sinensis & $409(226,183)$ & 86 & $318(77.75)$ & $308(96.86)$ & $295(95.78)$ & $171(57.97)$ & $124(42.03)$ \\
\hline$\left(\right.$ An. sinensis $\times$ An. kleini) $\mathrm{F}_{7} \times$ An. sinensis & $365(147,218)$ & 87 & $307(84.11)$ & $301(98.05)$ & $295(98.01)$ & $157(53.22)$ & $138(46.78)$ \\
\hline (An. sinensis $\times$ An. kleini) $\mathrm{F}_{8} \times$ An. sinensis & $355(154,201)$ & 84 & $284(80.00)$ & $281(98.94)$ & $267(95.02)$ & $139(52.06)$ & $128(47.94)$ \\
\hline (An. sinensis $\times$ An. kleini) $\mathrm{F}_{9} \times$ An. sinensis & $315(190,125)$ & 89 & $271(86.03)$ & $271(100.00)$ & $271(100.00)$ & $136(50.18)$ & $135(49.82)$ \\
\hline (An. sinensis $\times$ An. kleini) $\mathrm{F}_{10} \times$ An. sinensis & $336(162,174)$ & 94 & $291(86.61)$ & $266(91.40)$ & $257(96.62)$ & $126(49.03)$ & $131(50.97)$ \\
\hline$($ An. kleini $\times$ An. sinensis $) F_{1} \times$ An. kleini & $497(239,258)$ & 74 & $343(69.01)$ & $343(100.00)$ & $343(100.00)$ & $182(53.06)$ & $161(46.94)$ \\
\hline$($ An. kleini $\times$ An. sinensis $) \mathrm{F}_{2} \times$ An. kleini & $427(226,201)$ & 77 & $305(71.43)$ & $268(87.87)$ & $268(100.00)$ & $147(54.85)$ & $121(45.15)$ \\
\hline$($ An. kleini $\times$ An. sinensis $) F_{3} \times$ An. kleini & $421(209,212)$ & 70 & $286(67.93)$ & $257(89.86)$ & $257(100.00)$ & $126(49.03)$ & $131(50.97)$ \\
\hline$($ An. kleini $\times$ An. sinensis $) \mathrm{F}_{4} \times$ An. kleini & $458(211,247)$ & 79 & $328(66.00)$ & $302(92.07)$ & $287(95.03)$ & $155(54.01)$ & $132(45.99)$ \\
\hline \multicolumn{8}{|l|}{$F_{11}$ hybrid crosses } \\
\hline$\left[(\right.$ An. sinensis $\times$ An. kleini $) \mathrm{F}_{11} \times$ An. sinensis $]$ & $329(171,158)$ & 96 & $313(95.14)$ & $285(91.05)$ & $242(84.91)$ & $117(48.35)$ & $125(51.65)$ \\
\hline$\times\left[(A n\right.$. sinensis $\times$ An. kleini $) \mathrm{F}_{11} \times$ An. sinensis $]$ & & & & & & & \\
\hline
\end{tabular}

*Two selective egg-batches of inseminated females from each cross; ${ }^{\dagger}$ Dissection from 100 eggs; $n=$ number.

${ }^{+}$Sterile male hybrids with atrophy testes and accessory glands.

sinensis back crossing group, all the progenies were well matched An. sinensis NCBI sequences in the ITS2 sequence, except for 3 of $\mathrm{F}_{2}$-hybrid progenies (mixed). Also, all sequences of the COI gene showed $A n$. sinensis. In the (An. kleini $\times$ An. sinensis) $\times$ An. kleini backcrossing group, all sequences of the ITS2 showed An. kleini, but sequences of the COI gene matched An. kleini only in 3 of $\mathrm{F}_{2}$-hybrid progenies, and the remaining $\mathrm{F}_{3-5}$ progenies matched $A n$. sinensis (Table 1).

\section{Phylogenetic analysis}

The neighbor-joining (NJ) tree was constructed based on $\mathrm{COI}$ sequences in order to determine sequence divergence among the species examined (Table 1, Figure 2). The NJ tree showed concordant results with the PCR assay in all samples, which were divided into two major clades with 67-74\% bootstrap support. Clade I consisted of 55 samples of An. sinensis. It is interesting to note that among the 55 samples, 12 (KF0-2, KF0-3, KF0-4, KSKF3-1, KSKF3-2, KSKF3-3, KSKF4-1, KSKF4-2, KSKF4-3, KSKF5-1, KSKF5-
2 and KSKF5-3) and 8 (SKF1-1, SKF1-2, SKF1-3, KSF1-1, KSF1-3, SKSF2-1, SKSF2-2 and SKSF2-3), were identified as An. kleini and mixed sequences of both species, respectively, based on ITS2 sequences. Furthermore, these samples were placed within the same clade as the published sequence of An. sinensis (mean genetic distances = 0.003). Clade II comprised 5 samples of An. kleini derived from parental (KF0-1), reciprocal (KSF1-2) and backcrosses (KSKF2-1, KSKF2-2 and KSKF2-3) based on COI sequences (Table 1). The mean genetic distance between these two species was 0.023 .

\section{Pyrosequencing analysis}

The 24-nucleotide target region of the COI gene, including positions 237-260 of An. sinensis and An. kleini, was useful in classifying the sequenced species into 2 groups, as shown in Table 3 and Figures 3 and 4. The first group consisted of Parental: An. sinensis $\mathrm{F}_{0}$ (Figure 4A), $\mathrm{F}_{1}$ : An. sinensis x An. kleini - > hybrid $\mathrm{F}_{1}$ (Figure 4B), $\mathrm{F}_{5}$ : hybrid $\mathrm{F}_{4} \times$ An. sinensis $->$ hybrid $\mathrm{F}_{5}$ (Figure $4 \mathrm{C}$ ), $\mathrm{F}_{10}$ : hybrid $\mathrm{F}_{9}$ 

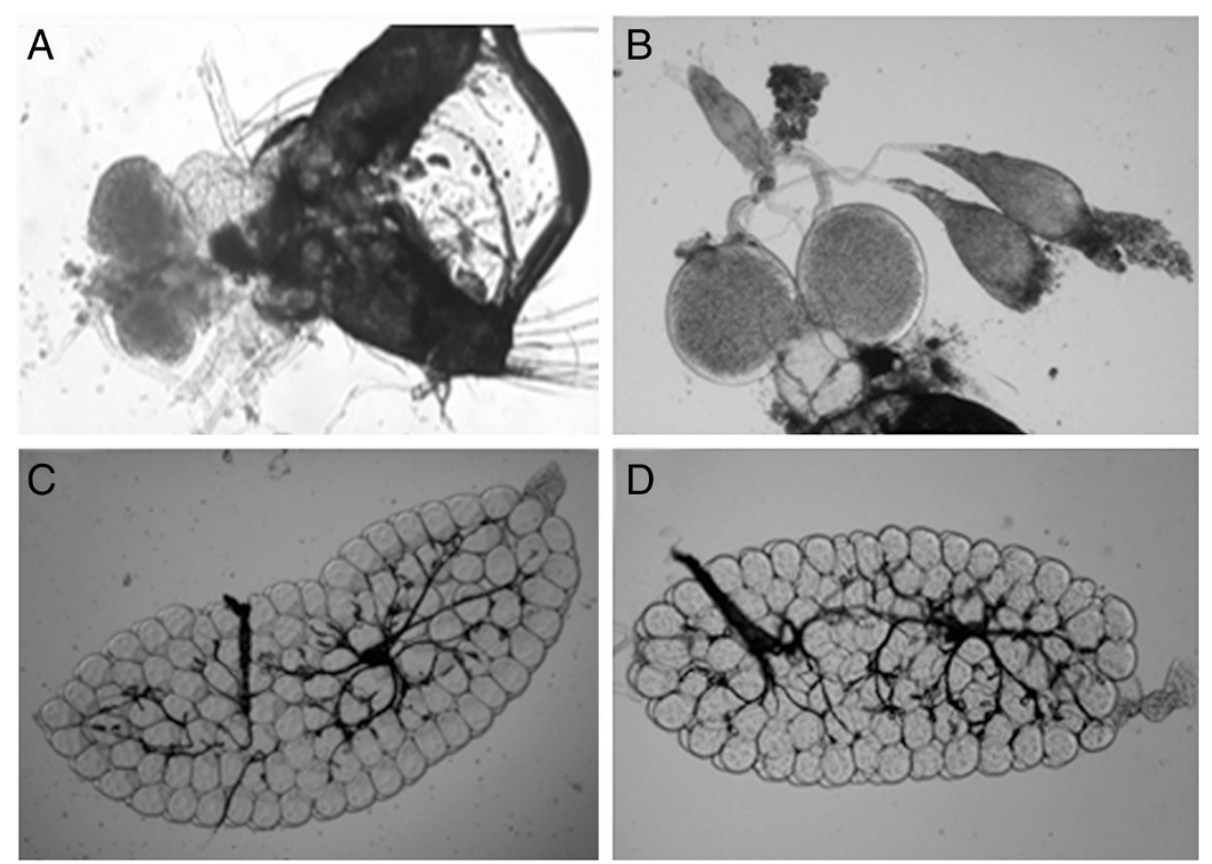

Figure 1 Reproductive system of adult males and females. (A) $F_{1}$-hybrid adult male of An. sinensis $x$ An. kleini showing atrophy of testes and accessory glands. (B) Backcross adult male of (An. kleini $\times$ An. sinensis) $F_{1} \times$ An. kleini showing normal development of testes and accessory glands. (C) $F_{1}$-hybrid adult female of An. sinensis $\times$ An. kleini showing normal development of ovarian follicles. (D) $F_{1}$-hybrid adult female of An. kleini $\times$ An. sinensis showing normal development of ovarian follicles.

$\mathrm{x}$ An. sinensis $->$ hybrid $\mathrm{F}_{10}$ (Figure $\left.4 \mathrm{D}\right)$, and $\mathrm{F}_{1}:$ An. kleini $\mathrm{x}$ An. sinensis - > hybrid $\mathrm{F}_{1}$ (Figure $4 \mathrm{~F}$ ) and another group comprised Parental: An. kleini $\mathrm{F}_{0}$ (Figure 4E) and $\mathrm{F}_{5}$ : hybrid $\mathrm{F}_{4} \mathrm{x}$ An. kleini - > hybrid $\mathrm{F}_{5}$ (Figure $4 \mathrm{G}$ ). Both groups differed from each other in three nucleotide positions (T237C, A243G and C253T) (Table 3). The positive-control plasmids showed similar results to the seven samples in the pyrogram, whereas, a negative control did not provide the pyrogram result.

\section{Discussion}

Introgression or introgressive hybridization is the movement of a gene (gene flow) between species through hybridization by repeated backcrossing of an interspecific hybrid with one of its parent species. It can have important effects on dynamics of the hybrid zone, speciation and adaptive radiation [41]. The variation of mitochondrial DNA is important and used widely for indirect studies of gene flow [42]. Thelwell et al. [43] reported evidence of mitochondrial (ND5) introgression between An. bwambae and An. gambiae. Consequently, extensive investigations of introgression between An. gambiae and An. arabiensis, and An. bwambae and An. gambiae have been documented systematically and extensively during the past decade [44-46]. Additionally, Walton et al. [42] demonstrated that mitochondrial DNA introgressed from An. baimaii (= dirus D) into An. dirus (=dirus A), and Morgan et al. [47] reported evidence to support the mitochondrial introgression between An. baimaii and An. dirus by the high levels of bidirectional mitochondrial gene flow detected between these 2 species. A large number of anopheline species were reported to be capable of interspecific hybridizations under laboratory conditions [48]. However, few species have succeeded in natural hybridization, for example, between An. gambiae and An. arabiensis [23], An. bwambae and An. gambiae [43], An. scanloni (= dirus C) and An. baimaii (= dirus D) [24], and An. minimus and An. harrisoni $[28,29]$. Recent discovery of the natural hybrid between $A n$. sinensis and An. kleini [27], and successful establishment of iso-female line colonies of these 2 anopheline species has urged this study to form a reliable systematic procedure to confirm this natural event and/or perform an introgressive study. The results of sterile $\mathrm{F}_{1}$-hybrid adult males with atrophy of accessory glands and testes obtained from the reciprocal crosses between these 2 anopheline species are in keeping with "Haldane Rule", which states that in interspecific crosses, the heterogametic sex $(\mathrm{X}, \mathrm{Y})$ will show sterility or viability problems before the homogametic sex [49]. Thus, the results of this study agree with those of Davidson [50], who reported that female and male hybrids obtained from An. gambiae and An. arabiensis were fertile and sterile, respectively. Furthermore, the results of this study are in accordance with crossing studies in the laboratory by Baimai et al. [51]. They demonstrated that $\mathrm{F}_{1}$ hybrids obtained from the cross of female An. dirus with male $A n$. scanloni were fertile and viable, with an exception of sterile 


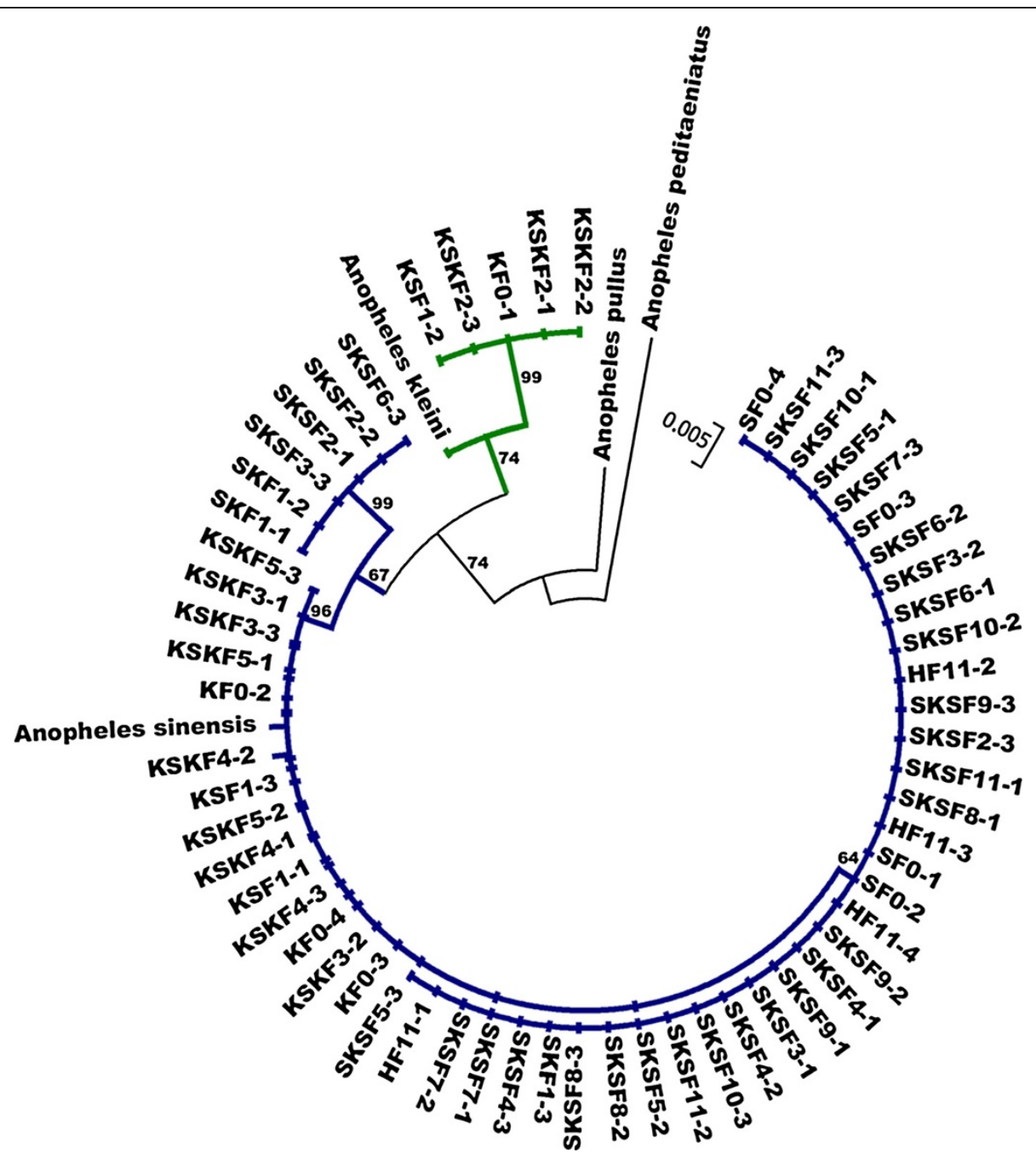

Figure 2 Neighbor-joining tree of An. sinensis and An. kleini based on COI sequences. Numbers on branches are bootstrap values (\%) of NJ analysis. Only greater than $50 \%$ bootstrap values are shown. Bars represent 0.005 substitutions per site. Detailed code of samples is shown in Table 1.

Table 3 Sample details and nucleotide positions used for discriminating between An. sinensis and An. kleini based on COI sequences

\begin{tabular}{|c|c|c|c|c|}
\hline \multirow[t]{2}{*}{ Code no. } & \multirow{2}{*}{$\begin{array}{l}\text { Samples } \\
\text { (Female x Male) }\end{array}$} & \multicolumn{3}{|c|}{ Nucleotide at position } \\
\hline & & 237 & 243 & 253 \\
\hline $\mathrm{A} 1^{*}$ & Parental: An. sinensis $\mathrm{F}_{0}$ & $T$ & A & $C$ \\
\hline $\mathrm{A} 2^{*}$ & $\mathrm{~F}_{1}$ : An. sinensis $\times$ An. kleini $->$ hybrid $\mathrm{F}_{1}$ & $\mathrm{~T}$ & A & C \\
\hline$A 3^{*}$ & $\mathrm{~F}_{5}$ : hybrid $\mathrm{F}_{4} \times$ An. sinensis $->$ hybrid $\mathrm{F}_{5}$ & $\mathrm{~T}$ & A & C \\
\hline $\mathrm{A} 4^{*}$ & $\mathrm{~F}_{10}$ : hybrid $\mathrm{F}_{9} \times$ An. sinensis $->$ hybrid $\mathrm{F}_{10}$ & $\mathrm{~T}$ & A & C \\
\hline $\mathrm{A} 5^{\dagger}$ & Parental: An. kleini $\mathrm{F}_{0}$ & C & G & $\mathrm{T}$ \\
\hline$A 6^{*}$ & $\mathrm{~F}_{1}$ : An. kleini $\times$ An. sinensis $->$ hybrid $\mathrm{F}_{1}$ & $\mathrm{~T}$ & A & C \\
\hline$A 7^{\dagger}$ & $\mathrm{F}_{5}$ : hybrid $\mathrm{F}_{4} \times$ An. kleini $->$ hybrid $\mathrm{F}_{5}$ & C & G & $\mathrm{T}$ \\
\hline
\end{tabular}




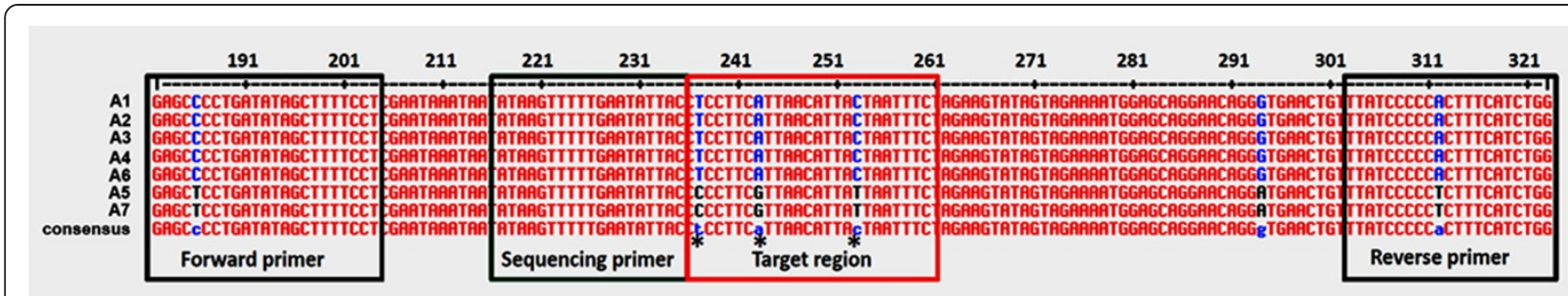

Figure 3 Alignment of the cytochrome $c$ oxidase subunit I (COI) gene derived from seven samples. Position of the forward primer (Anop_COI_F) and biotinylated reverse primer (Anop_COI_Rb) for template amplification are shown in the black boxes, while the sequencing primer (Anop_COI_S) and target region are shown in the green and red boxes, respectively. Asterisks indicate position of the target region used for species level identification.

males. It was interesting to note that the repeated backcross progenies in both directions, which resulted in obtaining $A n$. sinensis from hybrids of $\mathrm{F}_{2-11}$ progenies [(An. sinensis $\mathrm{x} A n$. kleini) $\mathrm{x} A n$. sinensis] and $\mathrm{F}_{3-5}$ progenies [(An. kleini x An. sinensis) x An. kleini], indicated the presence of introgressive hybridization between $A n$. sinensis and $A n$. kleini.

Regarding PCR identification of parental specimens, the exact species of An. sinensis and An. kleini were used in this study based on both the nuclear ITS2 and mitochondrial COI genes. Interestingly, 4 iso-female lines of $A n$. kleini showed the correct gene trait in the ITS2, but their COI sequences matched both species (i.e., An. kleini: 1 iso-female line, and An. sinensis: 3 iso-female lines). Therefore, the authors assumed that the COI sequences of $A n$. kleini had been replaced by those of $A n$. sinensis. Subsequently, reciprocal and repeated backcrosses were performed to clarify our hypothesis. Most COI sequence results of the progenies obtained from reciprocal and repeated backcrosses revealed that the mitochondrial COI gene introgressed from $A n$. sinensis into $A n$. kleini. This event resulted from introgression that occurred between these two species via consecutive repeated backcrosses. Thus, the sample identification of these 2 wild-caught species should be careful only when the COI barcoding region has been applied. Also, results from phylogenetic analysis confirmed the existence of an introgression phenomenon between them. Furthermore, the results from this study are in agreement with those of Petit and Excoffier [52]. They suggested that in species with male-biased dispersal (heterogametic sex), mtDNA markers should introgress more readily than nuclear ones.

Pyrosequencing is a unique sequencing method that was developed as an alternative to classical DNA sequencing for short- to medium-read applications. It is an accurate, simple and flexible bioluminometric method, which does not need labeled nucleotides or gel electrophoresis [53]. To date, this technology has been used successfully for high throughput identification of bacteria [54,55], virus $[56,57]$, protozoan parasites [53,58-60] and helminthes
[40]. This study applied the pyrosequencing technology for reliable identification from seven samples of $A n$. sinensis and An. kleini. The results were consistent with those of dideoxy sequencing and phylogentic analysis in six samples, except for the one (sample code no. A7) matched with An. kleini COI sequences. This sample possibly had a different mitochondrial haplotype from other hybrid progenies. More recently, the next-generation sequencing provided a good explanation of interspecific gene flow between An. gambiae and An. arabiensis [61] and An. gambiae $\mathrm{M}$ and $\mathrm{S}[62,63]$.

The effectiveness of a vector control strategy, and genetically modified strains of mosquitoes in a population that is unable to transmit malarial parasites, relies upon the gene flow within species and introgression [64]. Remarkably, Rheindt and Edwards [65] mentioned concern that in the long-term introgression with newcomer species may lead to a loss of genetic integrity in native species. Detection of natural hybridization is rare but meaningful in terms of horizontal transfer of advantageous genes, such as those in malaria susceptibility, particularly when species that allow hybridization are susceptible or refractory to malarial parasites $[9,66,67]$. This event is also involved in insecticide resistant genes, e.g., the $k d r$ gene [68,69] and ace-1 gene [70]. In addition, Morgan et al. [71] stated that the absence or presence of gene flow between populations and species has an impact on the dynamics of malaria transmission as well as construction of effective strategies for controlling malaria vectors. Our studies presented the introgressive events through consecutive repeated backcrosses under laboratory conditions, in which the mtDNA gene could be moved from one species to another. However, natural movement of the refractory gene to vivax malaria between sympatric populations of a low potential vector ( $A n$. sinensis) and a high potential vector $(A n$. kleini) needed intensive and systematic clarification. This new body of knowledge is anticipated to elucidate the promising strategies for replacing populations of high potential vectors with that of low potential vectors by using genetic manipulation through the gene introgression mechanism. 


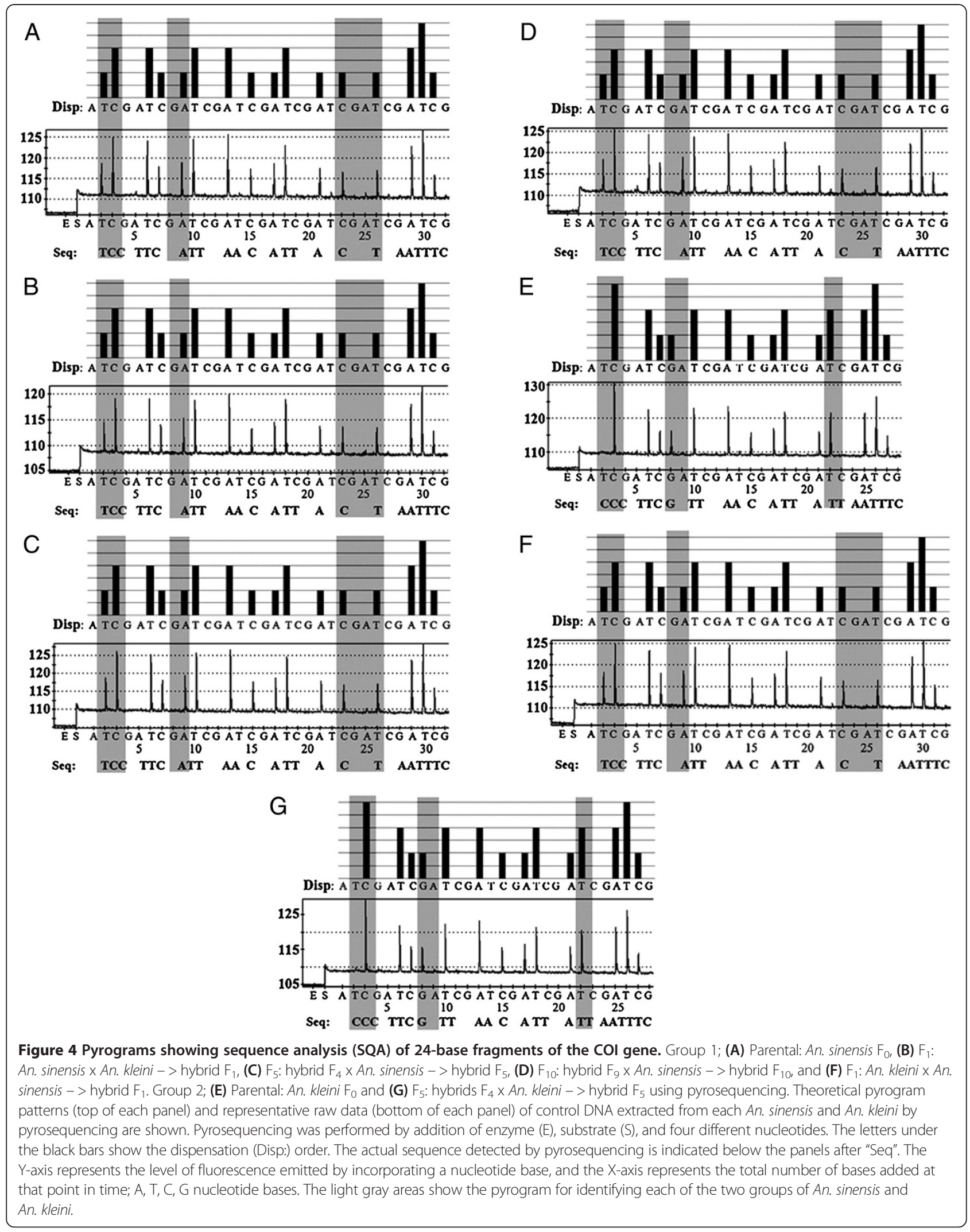




\section{Conclusions}

A single gravid, natural hybrid female between high ( $A n$. kleini) and low (An. sinensis) potential vectors of $P$. vivax was discovered in Paju City, Republic of Korea (ROK). The discovery of natural hybrids between these two anopheline species has led to systematic investigations of various aspects that clarify this event. Hybridization experiments used iso-female line colonies of these anophelines together with DNA analysis of ribosomal DNA [second internal transcribed spacer (ITS2)] and mitochondrial DNA [cytochrome $c$ oxidase subunit I (COI)] of the parental colonies, $\mathrm{F}_{1}$-hybrids and repeated backcross progenies were performed intensively by using a PCR-based assay and pyrosequencing technology. The results revealed that introgression of the COI gene between $A n$. sinensis and An. kleini was involved in this phenomenon. The pure An. sinensis obtained from hybrids of repeated backcross progenies in both directions, i.e., $\mathrm{F}_{2-11}$ progeny [(An. sinensis $\mathrm{x} A n$. kleini $) \times A n$. sinensis $]$ and $\mathrm{F}_{3-5}$ progeny [(An. kleini x An. sinensis) x An. kleini] provided obvious supportive evidence. The results emphasize a promising way to replace the population of a high potential vector $(A n$. kleini) with that of a low potential vector (An. sinensis) through the mechanism of gene introgression.

\section{Competing interests}

The authors declare that they have no competing interests.

\section{Authors' contributions}

All the authors contributed significantly to this study. WC and GSM designed the experiments, carried out field and laboratory experiments, interpreted the results, and wrote the manuscript. AS participated in hybridization experiments and molecular identifications. PMI, CT and VL carried out pyrosequencing analysis. All the authors read and approved the final version of the manuscript.

\section{Acknowledgements}

This work was supported financially by the National Research Council of Thailand (NRCT-NRF: NRF 1/2010 and NRF 1/2012) and Faculty of Medicine Research Fund, Chiang Mai University awarded to WC and AS, and the National Research Foundation of Korea (NRF) Grant (NRF-2010-C00033) awarded to GSM.

\section{Author details \\ 'Department of Parasitology, Faculty of Medicine, Chiang Mai University, Chiang Mai 50200, Thailand. '2Department of Biological Sciences, Inha University, Incheon 402-751, South Korea. ${ }^{3}$ Research and Diagnostic Center for Emerging Infectious Diseases, Khon Kaen University, Khon Kaen 40002, Thailand. ${ }^{4}$ Department of Parasitology, Faculty of Medicine, Khon Kaen University, Khon Kaen 40002, Thailand. 'Division of Cell Biology, Department of Preclinical Sciences, Faculty of Medicine, Thammasat University, Rangsit Campus, Pathum Thani 12121, Thailand. ${ }^{6}$ Department of Microbiology, Faculty of Medicine, Khon Kaen University, Khon Kaen 40002, Thailand.}

Received: 23 November 2013 Accepted: 18 January 2014 Published: 20 January 2014

\section{References}

1. Harbach RE: The phylogeny and classification of Anopheles. In Anopheles mosquitoes-new insights into malaria vectors. Edited by Manguin S. Croatia: InTech; 2013:3-55.
2. Harrison BA: A lectotype designation and description for Anopheles (An.) sinensis Wiedemann 1828, with a discussion of the classification and vector status of this and some other Oriental Anopheles. Mosq Syst 1973, 5:1-13.

3. Ree HI, Hwang UW, Lee IY, Kim TE: Daily survival and human blood index of Anopheles sinensis, the vector species of malaria in Korea. J Am Mosq Control Assoc 2001, 17:67-72.

4. Whang IJ, Jung J, Park JK, Min GS, Kim W: Intragenomic length variation of the ribosomal DNA intergenic spacer in a malaria vector, Anopheles sinensis. Mol Cells 2002, 14:158-162.

5. Ma Y, Xu J: The hyrcanus group of Anopheles (Anopheles) in China (Diptera: Culicidae): species discrimination and phylogenetic relationships inferred by ribosomal DNA internal transcribed spacer 2 sequences. J Med Entomol 2005, 42:610-619.

6. Lee WJ, Klein TA, Kim HC, Choi YM, Yoon SH, Chang KS, Chong ST, Lee IY, Jones JW, Jacobs JS, Sattabongkot J, Park JS: Anopheles kleini, Anopheles pullus, and Anopheles sinensis: potential vectors of Plasmodium vivax in the Republic of Korea. J Med Entomol 2007, 44:1086-1090.

7. Joshi D, Choochote W, Park MH, Kim JY, Kim TS, Suwonkerd W, Min GS: The susceptibility of Anopheles lesteri to infection with Korean strain Plasmodium vivax. Malar J 2009, 12(8):42.

8. Rueda LM, Li C, Kim HC, Klein TA, Foley DH, Wilkerson RC: Anopheles belenrae, a potential vector of Plasmodium vivax in the Republic of Korea. J Am Mosq Control Assoc 2010, 26:430-432.

9. Joshi D, Kim JY, Choochote W, Park MH, Min GS: Preliminary vivax malaria vector competence for three members of the Anopheles hyrcanus group in the Republic of Korea. J Am Mosq Control Assoc 2011, 27:312-314.

10. Sinka ME, Bangs MJ, Manguin S, Chareonviriyaphap T, Patil AP, Temperley WH, Gething PW, Elyazar IR, Kabaria CW, Harbach RE, Hay SI: The dominant Anopheles vectors of human malaria in the Asia-Pacific region: occurrence data, distribution maps and bionomic précis. Parasit Vectors 2011, 4:89.

11. Zhu G, Xia H, Zhou H, Li J, Lu F, Liu Y, Cao J, Gao Q, Sattabongkot J: Susceptibility of Anopheles sinensis to Plasmodium vivax in malarial outbreak areas of central China. Parasit Vectors 2013, 6:176.

12. Pan JY, Zhou SS, Zheng X, Huang F, Wang DQ, Shen YZ, Su YP, Zhou GC, Liu F, Jiang JJ: Vector capacity of Anopheles sinensis in malaria outbreak areas of central China. Parasit Vectors 2012, 5:136.

13. Zhou SS, Zhang SS, Wang JJ, Zheng X, Huang F, Li WD, Xu X, Zhang HW: Spatial correlation between malaria cases and water-bodies in Anopheles sinensis dominated areas of Huang-Huai plain, China. Parasit Vectors 2012, 5:106.

14. Makhawi AM, Liu XB, Yang SR, Liu QY: Genetic variations of ND5 gene of mtDNA in populations of Anopheles sinensis (Diptera: Culicidae) malaria vector in China. Parasit Vectors 2013, 6:290.

15. Sasa M: Human filariasis: A global survey of epidemiology and control. Tokyo: University of Tokyo Press; 1976.

16. Saeung A, Hempolchom C, Baimai V, Thongsahuan S, Taai K, Jariyapan N Chaithong U, Choochote W: Susceptibility of eight species members in the Anopheles hyrcanus group to nocturnally subperiodic Brugia malayi. Parasit Vectors 2013, 6:5.

17. Zhang HL: The natural infection rate of mosquitoes by Japanese encephalitis B virus in Yunnan Province. Zhonghua Yu Fang Yi Xue Za Zhi 1990, 24:265-267.

18. Kanojia PC, Shetty PS, Geevarghese G: A long-term study on vector abundance \& seasonal prevalence in relation to the occurrence of Japanese encephalitis in Gorakhpur district, Uttar Pradesh. Indian J Med Res 2003, 117:104-110.

19. Chai JY: Re-emerging Plasmodium vivax malaria in the Republic of Korea. Korean J Parasitol 1999, 37:129-143.

20. K-NIH: Korea-National Institute of Health: Manual for malaria control. Seoul: Korea: Korea-National Institute of Health (K-NIH); 2001.

21. Ree HI: Studies on Anopheles sinensis, the vector species of vivax malaria in Korea. In Today's malaria epidemiology and vector control proceedings of the 22nd seminar of tropical medicine: 21 February 2002; Seoul. Edited by Yong TS. South Korea: Yonsei Medical Center; 2002:33-52.

22. K-CDC: Korea-Center for Disease Control and Prevention: Provisional cases of selected notifiable disease. K-CDC Communicable Disease Weekly Report (CDWR): 25-31 December 2005, Volume 53. :1-32.

23. Temu EA, Hunt RH, Coetzee M, Minjas JN, Shiff CJ: Detection of hybrids in natural populations of the Anopheles gambiae complex by the rDNAbased, PCR method. Ann Trop Med Parasitol 1997, 91:963-965.

24. Walton C, Sharpe RG, Pritchard SJ, Thelwell NJ, Butlin RK: Molecular identification of mosquito species. Biol J Linn Soc 1999, 68:241-256. 
25. Van Bortel W, Trung HD, Roelants P, Harbach RE, Backeljau T, Coosemans M: Molecular identification of Anopheles minimus s.l. beyond distinguishing the members of the species complex. Insect Mol Biol 2000, 9:335-340.

26. Kengne $P$, Trung HD, Baimai V, Coosemans M, Manguin S: A multiplex PCRbased method derived from random amplified polymorphic DNA (RAPD) markers for the identification of species of the Anopheles minimus group in Southeast Asia. Insect Mol Biol 2001, 10:427-435.

27. Joshi D, Choochote W, Min GS: Short report: natural hybrid between Anopheles kleini and Anopheles sinensis. Am J Trop Med Hyg 2009, 81:1020-1022.

28. Choochote W, Saeung A: Systematic techniques for the recognition of Anopheles species complexes. In Anopheles mosquitoes-new insights into malaria vectors. Edited by Manguin S. Croatia: InTech; 2013:57-79.

29. Tanaka K, Mizusawa K, Saugstad ES: A revision of the adult and larval mosquitoes of Japan (including the Ryukyu Archipelago and the Ogasawara Islands) and Korea (Diptera: Culicidae). Contrib Am Entomol Inst 1979, 16:1-985.

30. Joshi D, Park MH, Saeung A, Choochote W, Min GS: Multiplex assay to identify Korean vectors of malaria. Mol Ecol Resour 2010, 10:748-750.

31. Saeung A, Otsuka $Y$, Baimai $V$, Somboon P, Pitasawat B, Tuetun B, Junkum A, Takaoka H, Choochote W: Cytogenetic and molecular evidence for two species in the Anopheles barbirostris complex (Diptera: Culicidae) in Thailand. Parasitol Res 2007, 101:1337-1344.

32. Li C, Lee JS, Groebner JL, Kim HC, Klein T, O'Guinn ML, Wilkerson RC: A newly recognized species in the Anopheles hyrcanus group and molecular identification of related species from the Republic of South Korea (Diptera: Culicidae). Zootaxa 2005, 939:1-8.

33. Folmer O, Black M, Hoeh W, Lutz R, Vrijenhoek R: DNA primers for amplification of mitochondrial cytochrome $c$ oxidase subunit I from diverse metazoan invertebrates. Mol Mar Biol Biotechnol 1994, 3:294-299.

34. Hall TA: BioEdit: a user-friendly biological sequence alignment editor and analysis program for Windows 95/98/NT. Nucleic Acids Symp Ser 1999, 41:95-98.

35. Thompson JD, Higgins DG, Gibson TJ: CLUSTAL W: improving the sensitivity of progressive multiple sequence alignment through sequence weighting, positions-specific gap penalties and weight matrix choice. Nucleic Acids Res 1994, 22:4673-4680.

36. Saitou N, Nei M: The neighbor-joining method: a new method for reconstructing phylogenetic trees. Mol Biol Evol 1987, 4:406-425.

37. Tamura K, Dudley J, Nei M, Kumar S: MEGA4: Molecular evolution genetics analysis (MEGA) software version 4.0. Mol Biol Evol 2007, 24:1596-1599.

38. Choochote W: Evidence to support karyotypic variation of the mosquito, Anopheles peditaeniatus in Thailand. J Insect Sci 2011, 11:10.

39. Park SJ, Choochote W, Jitpakdi A, Junkum A, Kim SJ, Jariyapan N: Evidence for a conspecific relationship between two morphologically and cytologically different forms of Korean Anopheles pullus mosquito. Mol Cells 2003, 16:354-360.

40. Tantrawatpan C, Intapan PM, Janwan P, Sanpool O, Lulitanond V, Srichantaratsamee C, Anamnart W, Maleewong W: Molecular identification of Paragonimus species by DNA pyrosequencing technology. Parasitol Int 2013, 62:341-345.

41. Grant PR, Grant BR, Petren K: Hybridization in the recent past. Am Nat 2005, 166:56-67

42. Walton C, Handley JM, Tun-Lin W, Collins FH, Harbach RE, Baimai V, Butlin RK: Population structure and population history of Anopheles dirus mosquitoes in Southeast Asia. Mol Biol Evol 2000, 17:962-974.

43. Thelwell NJ, Huisman RA, Harbach RE, Butlin RK: Evidence for mitochondrial introgression between Anopheles bwambae and Anopheles gambiae. Insect Mol Biol 2000, 9:203-210.

44. Besansky NJ, Krzywinski J, Lehmann T, Simard F, Kern M, Mukabayire O, Fontenille D, Touré $Y$, Sagnon N: Semipermeable species boundaries between Anopheles gambiae and Anopheles arabiensis: evidence from multilocus DNA sequence variation. Proc Natl Acad Sci USA 2003, 100:10818-10823.

45. Slotman M, Della Torre A, Powell JR: Female sterility in hybrids between Anopheles gambiae and A. arabiensis, and the causes of Haldane's rule. Evolution 2005, 59:1016-1026.

46. Slotman MA, Della Torre A, Calzetta M, Powell JR: Differential introgression of chromsomal regions between Anopheles gambiae and An. arabiensis. Am J Trop Med Hyg 2005, 73:326-335.
47. Morgan K, Linton YM, Somboon P, Saikia P, Dev V, Socheat D, Walton C: Inter-specific gene flow dynamics during the Pleistocene-dated speciation of forest-dependent mosquitoes in Southeast Asia. Mol Ecol 2010, 19:2269-2285.

48. Presgraves DC, Orr HA: Haldane's rule in taxa lacking a hemizygous X. Science 1998, 282:952-954.

49. Orr HA: Haldane's rule. Annu Rev Ecol Evol Syst 1997, 28:195-218.

50. Davidson G: The five mating-types in the Anopheles gambiae complex. Riv Malariol 1964, 43:167-183.

51. Baimai $V$, Andre RG, Harrison BA, Kijchalao U, Panthusiri L: Crossing and chromosomal evidence for two additional sibling species within the taxon Anopheles dirus Peyton and Harrison (Diptera: Culicidae) in Thailand. Proc Entomol Soc Wash 1987, 89:157-166.

52. Petit RJ, Excoffier L: Gene flow and species delimitation. Trends Ecol Evol 2009, 24:386-393.

53. Sreekumar C, Hill DE, Miska KB, Vianna MC, Yan L, Myers RL, Dubey JP: Genotyping and detection of multiple infections of Toxoplasma gondii using Pyrosequencing. Int J Parasitol 2005, 35:991-999.

54. Monstein H, Nikpour-Badr S, Jonasson J: Rapid molecular identification and subtyping of Helicobacter pylori by pyrosequencing of the 16S rDNA variable V1 and V3 regions. FEMS Microbiol Lett 2001, 199:103-107.

55. Wahab T, Hjalmarsson S, Wollin R, Engstrand L: Pyrosequencing Bacillus anthracis. Emerg Infect Dis 2005, 11:1527-1531.

56. O'Meara D, Wilbe K, Leitner T, Hejdeman B, Albert J, Lundeberg J: Monitoring resistance to human immunodeficiency virus type 1 protease inhibitors by pyrosequencing. J Clin Microbiol 2001, 39:464-473.

57. Elahi E, Pourmand N, Chaung R, Rofoogaran A, Boisver J, Samimi-Rad K, Davis RW, Ronaghi M: Determination of hepatitis C virus genotype by Pyrosequencing. J Virol Methods 2003, 109:171-176.

58. Stensvold CR, Traub RJ, von Samson-Himmelstjerna G, Jespersgaard C, Nielsen HV, Thompson RC: Blastocystis: subtyping isolates using pyrosequencing technology. Exp Parasitol 2007, 116:111-119.

59. Stensvold CR, Lebbad M, Verweij JJ, Jespersgaard C, von SamsonHimmelstjerna G, Nielsen SS, Nielsen HV: Identification and delineation of members of the Entamoeba complex by pyrosequencing. Mol Cell Probes 2010, 24:403-406.

60. Lulitanond V, Intapan PM, Tantrawatpan C, Sankuntaw N, Sanpool O, Janwan P, Maleewong W: Molecular markers for detection and differentiation of Plasmodium falciparum and Plasmodium vivax in human blood samples by pyrosequencing. J Clin Microbiol 2012, 50:1455-1457.

61. Neafsey DE, Lawniczak MK, Park DJ, Redmond SN, Coulibaly MB, Traoré SF Sagnon N, Costantini C, Johnson C, Wiegand RC, Collins FH, Lander ES, Wirth DF, Kafatos FC, Besansky NJ, Christophides GK, Muskavitch MA: SNP genotyping defines complex gene-flow boundaries among African malaria vector mosquitoes. Science 2010, 330:514-517.

62. Reidenbach KR, Neafsey DE, Costantini C, Sagnon N, Simard F, Ragland GJ, Egan SP, Feder JL, Muskavitch MA, Besansky NJ: Patterns of genomic differentiation between ecologically differentiated $\mathrm{M}$ and $\mathrm{S}$ forms of Anopheles gambiae in West and Central Africa. Genome Biol Evol 2012, 4:1202-1212.

63. Weetman D, Wilding CS, Steen K, Pinto J, Donnelly MJ: Gene flow-dependent genomic divergence between Anopheles gambiae $\mathrm{M}$ and $\mathrm{S}$ forms. Mol Biol Evol 2012, 29:279-291

64. Curtis C: The case for malaria control by genetic manipulation of its vectors. Parasitol Today 1994, 10:371-374.

65. Rheindt FE, Edwards SV: Genetic introgression: an integral but neglected component of speciation in birds. Auk 2011, 128:620-632.

66. Rottschaefer SM, Riehle MM, Coulibaly B, Sacko M, Niaré O, Morlais I, Traoré SF, Vernick KD, Lazzaro BP: Exceptional diversity, maintenance of polymorphism, and recent directional selection on the APL1 malaria resistance genes of Anopheles gambiae. PLoS Biol 2011, 9:e1000600.

67. White BJ, Lawniczak MK, Cheng C, Coulibaly MB, Wilson MD, Sagnon N, Costantini C, Simard F, Christophides GK, Besansky NJ: Adaptive divergence between incipient species of Anopheles gambiae increases resistance to Plasmodium. Proc Natl Acad Sci USA 2011, 108:244-249.

68. Weill M, Chandre F, Brengues C, Manguin S, Akogbeto M, Pasteur N, Guillet P, Raymond M: The kdr mutation occurs in the Mopti form of Anopheles gambiae s.s. through introgression. Insect Mol Biol 2000, 9:451-455.

69. Diabate A, Baldet T, Chandre C, Dabire KR, Kengne P, Guiguemde TR, Simard F, Guillet P, Hemingway J, Hougard JM: KDR mutation, a genetic 
marker to assess events of introgression between the molecular $M$ and $\mathrm{S}$ forms of Anopheles gambiae (Diptera: Culicidae) in the tropical savannah area of West Africa. J Med Entomol 2003, 40:195-198.

70. Djogbenou L, Chandre F, Berthomieu A, Dabire R, Koffi A, Alout H, Weill M: Evidence of introgression of the ace-1(R) mutation and of the ace-1 duplication in West African Anopheles gambiae s.s. PLoS One 2008, 3:e2172.

71. Morgan K, Somboon P, Walton C: Understanding Anopheles diversity in Southeast Asia and its applications for malaria control. In Anopheles mosquitoes-new insights into malaria vectors. Edited by Manguin S. Croatia: InTech; 2013:327-355.

doi:10.1186/1756-3305-7-36

Cite this article as: Choochote et al.: Evidence to support natural hybridization between Anopheles sinensis and Anopheles kleini (Diptera: Culicidae): possibly a significant mechanism for gene introgression in sympatric populations. Parasites \& Vectors 2014 7:36.

\section{Submit your next manuscript to BioMed Central and take full advantage of:}

- Convenient online submission

- Thorough peer review

- No space constraints or color figure charges

- Immediate publication on acceptance

- Inclusion in PubMed, CAS, Scopus and Google Scholar

- Research which is freely available for redistribution 